Chirurg 2014 · 85:347

DOI 10.1007/s00104-014-2726-x

Online publiziert: 12. März 2014

๑) Springer-Verlag Berlin Heidelberg 2014

H. Dralle $\cdot$ I. Satiroglu

Universitätsklinik für Allgemein-, Viszeral- und Gefäßchirurgie,

Medizinische Fakultät, Universitätsklinikum Halle (Saale)

\title{
Endoskopische Resektion von Typ-1-Magenkarzinoiden
}

Einverständnis für eine EMR oder Operation, sie wurden nicht in die Studie eingeschlossen.

\section{Ergebnisse}

Im Zeitraum der Studie wurden 27.000 proximale Intestinoskopien durchgeführt, bei 38 Patienten wurde ein Magen-NEN, bei 27 Patienten ein Typ-1-NEN (0,1\%) verifiziert und bei 22 Patienten durch EMR mit tumorfreien Resektionsrändern entfernt. Fünf der 22 in die Studie eingeschlossenen Patienten (23\%) hatten ein singuläres, 17 multiple (2-5) Typ-1-NEN. Die Tumorgröße war bei 18 Patienten 5$10 \mathrm{~mm}$, bei 4 Patienten $10-20 \mathrm{~mm}$. Der mittlere Ki67-Index betrug 3,7\% (1-11\%). Bei einem Patienten trat eine EMR-bedingte Perforation auf und erforderte eine chirurgische Intervention.

Vier Patienten (18\%) hatten nach 12 bis 18 Monaten Rezidive (2 Patienten 2-malig), die wiederum durch EMR entfernt wurden. Die Gastrinspiegel nach EMR lagen im Verlauf unter 300 pg/ml. Die Überlebens- und Krankheitsfreiheitsraten waren $100 \%$.

\section{Methoden}

Zwischen 1999 und 2012 wurden $22 \mathrm{~Pa}$ tienten mit Magen-NEN durch EMR behandelt und im Median 7 Jahre nachbeobachtet. Die endoskopische Intervention schloss bei allen Patienten eine Chromoendoskopie mit Methylenblau ein, alle im Korpus-Fundus-Bereich identifizierten Läsionen wurden entfernt. Alle Patienten wurden postinterventionell in 6-monatigem Abstand kontrolliert (proximale Intestinoskopie, Ultraschall, Gastrinbestimmung). Fünf Patienten gaben kein

\section{Diskussion und Fazit}

Vorteil der vorliegenden Studie ist die lange Nachbeobachtungszeit von im Median 7 Jahren, bislang die längste Nachbeobachtungszeit vergleichbarer Studien. Zwischen der Anzahl der Typ-1-NEN und ihrem Durchmesser bestand hinsichtlich der Rezidivrate keine Korrelation. Aus chirurgischer Sicht ergibt sich daher schlussfolgernd keine Indikation zum operativ-resezierenden Vorgehen (z. B. Antrektomie) bei mono- oder oligofokalen Typ-1-NEN der Größe bis $20 \mathrm{~mm}$. Da bei allen in die Studie eingeschlossenen Patienten die NEN durch EMR vollständig entfernt wurden, konnte die Chirurgen nicht selten gestellte Frage des weiteren Vorgehens nach unvollständiger EMR nicht geklärt werden. Aus anderen Studien mit endoskopischem Waitand-see-Vorgehen ist jedoch zu schließen, dass zumindest bei Typ-1-NEN $<10 \mathrm{~mm}$ eine chirurgische Nachresektion nicht erforderlich ist [1]. Alternativ käme dabei auch eine medikamentöse Therapie mit Somatostatin oder dem Gastrinrezeptorantagonist Netazepid infrage $[2,3]$.

\section{Korrespondenzadresse}

Prof. Dr. Dr. h.c. H. Dralle

Universitätsklinik für Allgemein-, Viszeralund Gefäßchirurgie, Medizinische Fakultät, Universitätsklinikum Halle, Ernst-Grube-Str. 40, 06097 Halle (Saale) henning.dralle@uk-halle.de

Interessenkonflikt. H. Dralle und I. Satiroglu geben an, dass kein Interessenkonflikt besteht.

\section{Literatur}

1. Ravizaa D, Fiori G, Trovato C et al (2007) Long-term endoscopic and clincial follow-up of untreated type 1 gastric neuroendocrine tumours. Dig Liver Dis 39:537-543

2. Fykse V, Sandvik AK, Qvigstad G et al (2004) Treatment of ECL cell carcinoids with octreotide LAR. Scand J Gastroenterol 39:621-628

3. Fossmark R, Sordal O, Jianu CS et al (2012) Treatment of gastric carcinoids type 1 with the gastrin receptor antagonist netazepide (YF476) results in regression of tumours and normalisation of serum chromogranin A. Aliment Pharmacol Ther 36:1067-1075 